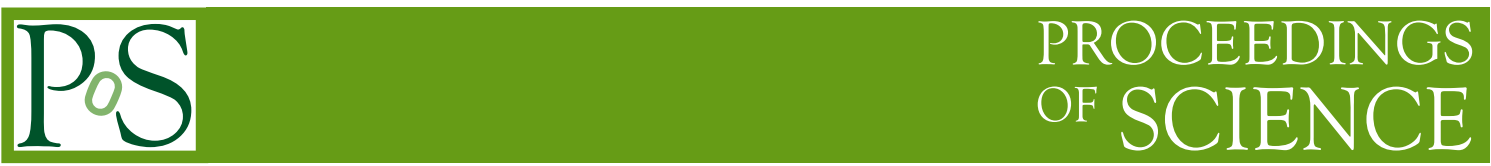

\title{
Relativistic jet feedback in galaxy formation
}

\section{Geoffrey V. Bicknell*}

Research School of Astronomy \& Astrophysics, Australian National University

E-mail: geoffemso.anu.edu.au

\section{Alexander Y. Wagner}

Research School of Astronomy \& Astrophysics, Australian National University

E-mail: ayw@mso.anu.edu.au

Galaxies grow through the hierarchical merging of smaller galaxies. However, the bright part of the galaxy luminosity function is incompatible with the simplest version of hierarchical merging. Feedback from the central black hole in the host galaxies may reduce the number of bright galaxies and regulate the co-evolution of black hole and host galaxy. Simulations of galaxy evolution have attempted to include the physical effects of AGN feedback with a resolution usually exceeding a few kiloparsecs. However, interactions between jets and the interstellar medium naturally involve processes occurring on less than kiloparsec scales. In order to understand the processes occurring on such scales, we present a comprehensive suite of simulations of relativistic jets interacting with a fractal two-phase interstellar medium with a resolution of two parsecs and a largest scale of one kiloparsec. The transfer of energy and momentum to the interstellar medium is considerable, and we find that jets with powers in the range of $10^{43}-10^{46} \mathrm{ergs} \mathrm{s}^{-1}$ can inhibit star formation through the dispersal of dense gas in the galaxy core. We also present results quantitatively showing the effectiveness of jet-mediated feedback as a function of the ratio of the jet power to the Eddington luminosity of the black hole, the pressure of the interstellar medium and the porosity of the dense gas.

25th Texas Symposium on Relativistic Astrophysics - TEXAS 2010

December 06-10, 2010

Heidelberg, Germany

\footnotetext{
* Speaker.
} 


\section{Issues in galaxy formation}

We know that

1. Hierarchical merging predicts more high mass galaxies than are observed; this is manifest in the exponential cutoff in the commonly used Schechter luminosity function.

2. The shape of the galaxy luminosity function apparently requires some form of feedback in addition to that provided by supernovae and this feedback regulates star formation.

3. This leads to the concept of downsizing: Star formation and AGN activity takes place more vigorously and in higher mass objects at $z \sim 1-2$ and subsequently in lower mass objects for $z \lesssim 1$.

A good discussion of these issues is in [1] in which semi-analytic models were used to estimate the effect of "radio-mode" feedback on galaxy formation. Somewhat surprisingly they found that feedback could be effective if the accretion rate onto the central black hole were orders of magnitude below the Eddington rate. This implies that low-powered (Fanaroff-Riley Class I) radio galaxies are providing the feedback. However, as we show in the following, this conclusion can be attributed to their assumption of a smooth low density interstellar medium - similar in some respects to what we see now in clusters of galaxies where significant feedback in the form of heating of the intra-cluster medium is known to occur.

We expect that in newly forming galaxies the distribution of gas near the core would have been dense and inhomogeneous. This is indicated, for example, by cosmological SPH simulations [2]. In order to achieve a satisfactory Bondi accretion rate onto the central black hole, [2] assumes an increase in density by approximately a factor of 100 , in the core relative to the kpc scale, which is the resolution of their simulations.

For these reasons, we have been conducting high resolution simulations of the cores of elliptical galaxies in which the central kiloparsec consists of fractal two-phase interstellar medium through which a radio jet propagates [see 3, 4, 5]. Our largest scale in the simulations that we present here, corresponds to the resolution of current cosmological simulations; our grid size is effectively $512^{3}$ (using a FLASH adaptive mesh grid) so that our smallest scale is two parsecs. This allows us to adequately resolve much of the physics that is related to AGN feedback.

\section{GPS and CSS radio sources}

When discussing radio mode feedback on kpc scales, it is not widely appreciated that the related radio sources are the Gigahertz Peak Spectrum (GPS) and Compact Steep Spectrum (CSS) sources representing about $25 \%$ of sources in flux density limited catalogues as well as the High Redshift Radio Galaxies (HzRGs). In all of these sources there is ample evidence for strong interaction with the interstellar medium. They represent radio-mode feedback in action. Moreover, any model of radio-mode feedback not only needs to account for the optical luminosity function of galaxies but also the morphological appearance and optical emission line properties of these objects. 
GPS sources are typically of order $500 \mathrm{pc}$ in size and have an inverted radio spectrum below about a GHz, which has been interpreted as the result of free-free absorption in the surrounding gas [6] or synchrotron self-absorption [7]. The CSS sources typically have spectra which turn over at about $100 \mathrm{MHz}$ and are generally of order $5 \mathrm{kpc}$ in size. It is generally agreed that GPS and CSS sources represent different evolutionary stages of the same sort of object - young radio galaxies.

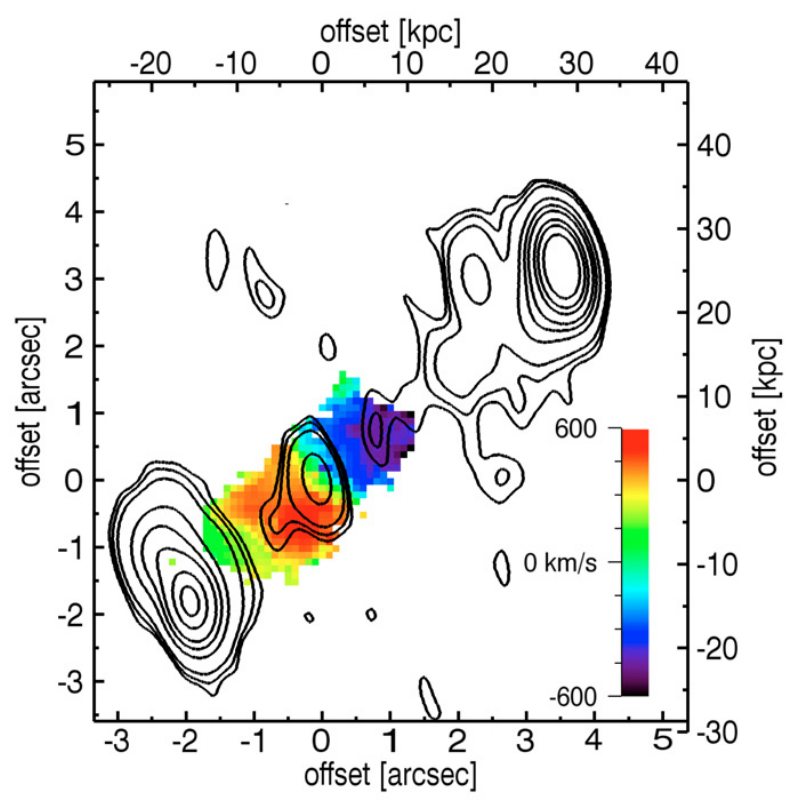

Figure 1: Radio contours of MRC 0406-244 overlaid on an [OIII] velocity image [8]. This image clearly shows the blueshifted and redshifted [OIII] emission produced by the interaction of the powerful radio source with clouds in the central region of the galaxy.

HZRGs are found at high redshifts by virtue of their steep radio spectra and interact strongly withe emission line gas surrounding them. An example from [8] is shown in Figure 1 and that image shows very clearly the disruption of line emitting gas, which is dense relative to the hot atmosphere of the galaxy and which is being driven outwards by the radio plasma. Note also the containment of some of the radio source within the emission line region.

\section{Simulations}

\subsection{Parameters}

Figure 2 is a snapshot from one of our simulations, which summarises the parameters and their ranges as well as the type of two-phase medium which we employ. The inclusion of a twophase medium is crucial. This provides an obstacle through which the jets propagate while at the same time the porosity of the medium provides an escape route for the relativistic plasma. The net result is a "flood and channel" phase in the early history of a radio galaxy, which isotropises the initially well-collimated jet. Details of the mathematical construction of the two phase medium are provided in [4]. 


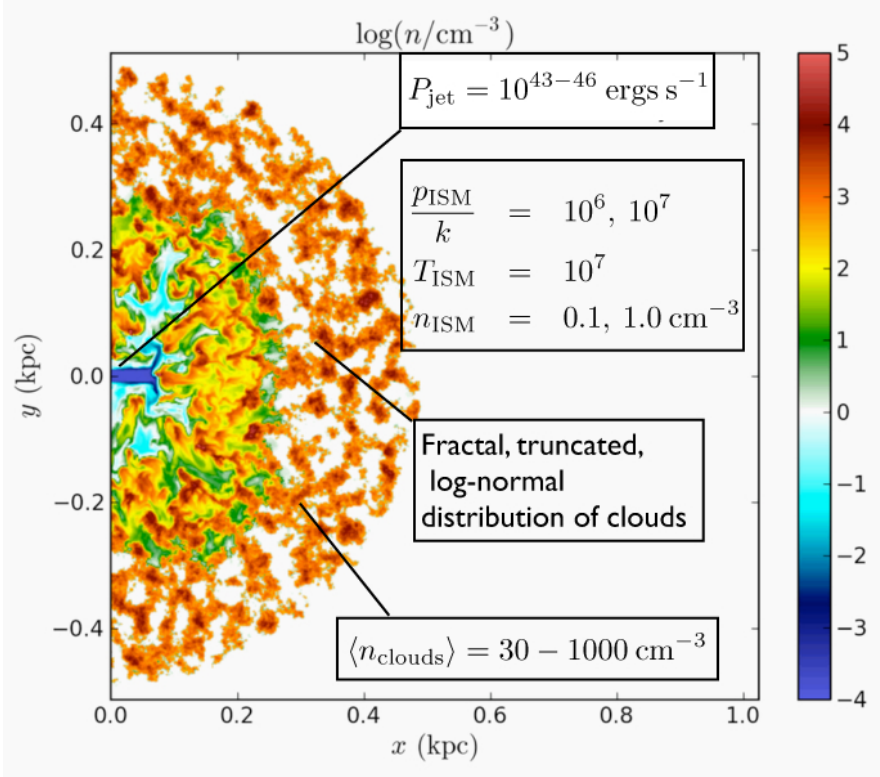

Figure 2: This snapshot from a simulation summarises the parameters and ISM model used in the simulations.

\subsection{Jet-inhomogeneous ISM interaction}

Figure 3 shows the progress of a $10^{45} \mathrm{ergs} \mathrm{s}^{-1}$ jet through a clumpy medium in which the average warm $\left(T=10^{4} \mathrm{~K}\right.$ ) cloud density is $100 \mathrm{~cm}^{-3}$ (Simulation E; see Fig, 5). The filling factor of the warm clouds is 0.42 and their total mass is $1.6 \times 10^{9} \mathrm{M} \odot$.

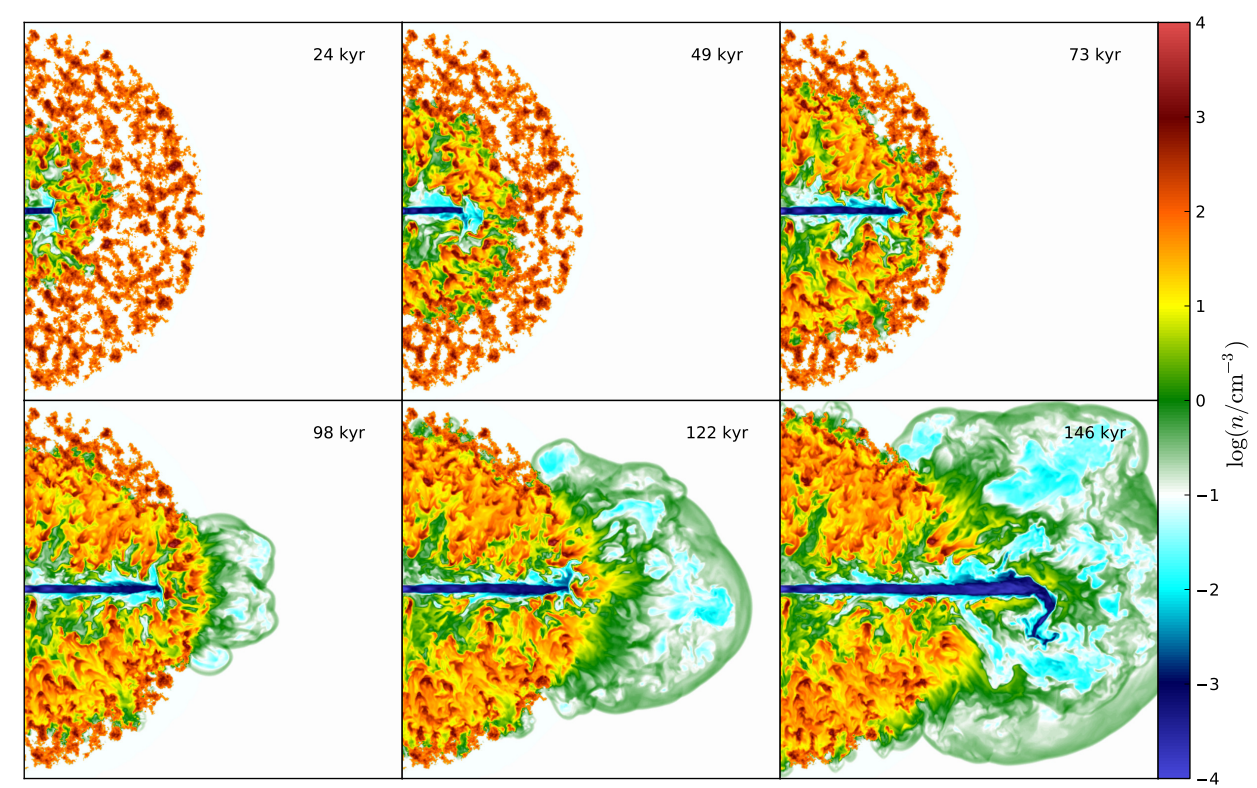

Figure 3: Progress of a $10^{45} \mathrm{ergs} \mathrm{s}^{-1}$ jet through a clumpy medium (Simulation E). The density scale is logarithmic. 


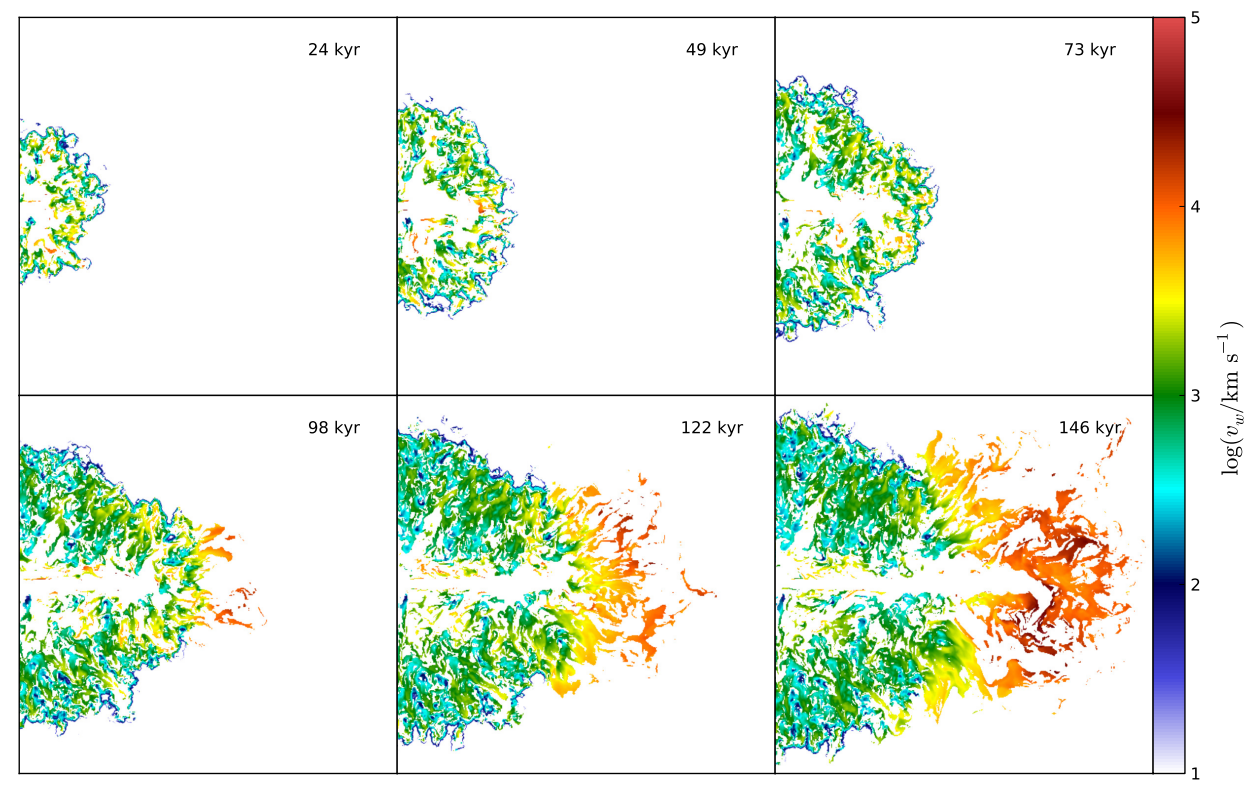

Figure 4: The radial velocity field at various stages in the evolution of simulation E. The colour scale is logarithmic.

There are two main stages in the morphology of the radio galaxy revealed by these images. Initially the jet is deflected in numerous directions by the obstructing clouds, At the same time the shocked jet plasma expands within the cloud region forming an expanding bubble; the warm clouds are driven outwards by the expansion of this high pressured bubble. In Simulation E, for example, Figure 4 shows cloud velocities ranging from a few hundred to a thousand kilometres per second. (There are even larger velocities, which result from a small admixture of cloud material into the relativistic plasma.)

The clouds would emit a characteristic shock excited spectrum possibly mixed with some photoionisation by the nucleus. At about $t=98 \mathrm{kyr}$ the jet bursts free and forms an expanding radio lobe, which is now only confined by the hot interstellar medium. At late stages the morphology of the radio source starts to resemble the image of thehigh redshift radio galaxy shown in Figure 1 from [8]. Two features, in particular, are worth noting: (1) The expanding radio lobe beyond the emission line gas (2) The confinement of some radio plasma within the emission line region.

\section{Results of parameter study}

Figure 5 summarises our extensive parameter study of jet-ISM interactions. The mean radial velocity of clouds affected by the jet is plotted against jet power. For comparison the dashed lines represent the loci of velocity dispersion versus jet power for given ratios, $\eta$, of jet power to Eddington luminosity [see 5, for details]. We adopt the criterion that a jet will disperse clouds when the mean radial velocity of clouds exceeds the velocity dispersion of the host. Hence, for a given value of $\eta$, if a simulation point lies above the corresponding dashed line then the criterion is satisfied. For example, if $\eta=10^{-3}$ then the jet in simulation $\mathrm{E}$ is sufficiently powerful to disperse the clouds and in simulation $\mathrm{D}$ the result is marginal. The jet in $\mathrm{D}^{\prime}$ would not disperse the more 


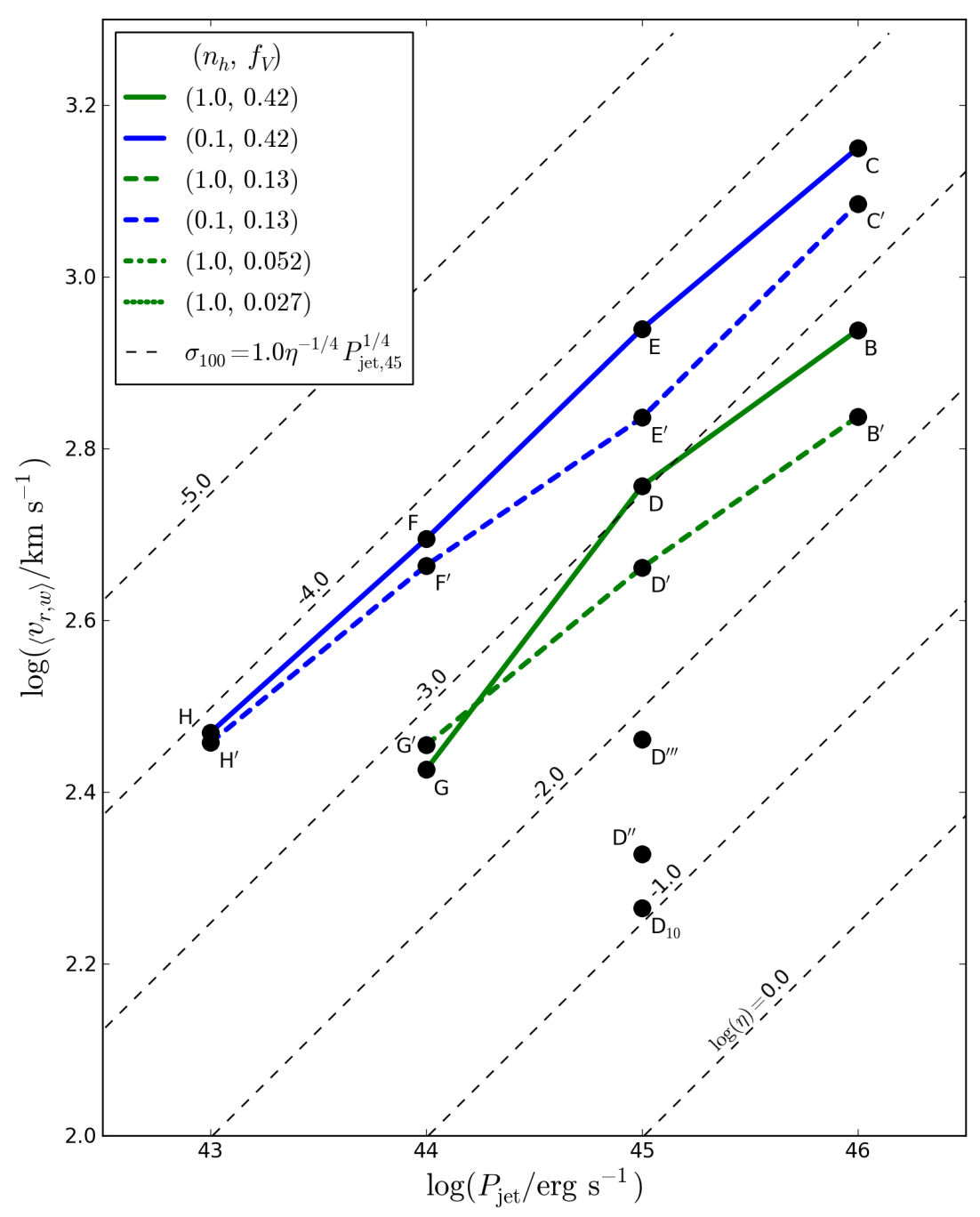

Figure 5: The points represent the jet power and mean radial velocity of clouds in various simulations.

porous cloud distribution. As a general rule the more porous distributions of clouds require a higher jet power in order to disperse the clouds. This reflects that fact that the jet can escape more easily when the cloud distribution is more porous. It is also evident from this figure that for velocity dispersions in excess of $200 \mathrm{~km} \mathrm{~s}^{-1}$, jets with powers above $10^{43} \mathrm{ergs} \mathrm{s}^{-1}$ can contribute to the removal of gas from the core.

Note that some points additional to those presented in [5], viz. $\mathrm{D}^{\prime \prime}, \mathrm{D}^{\prime \prime \prime}$ and $D_{10}$, are included in Figure 5. The first two points represent higher porosity and the third point corresponds to a lower value for the wavenumber cutoff in the distribution of fractal clouds (i.e. larger clouds). More details will be presented in a future paper.

\section{Concluding remarks}

A recent paper [9] is at least consistent with the picture presented here. Kormendy and col- 
leagues find that ellipticals with $-21.54<M_{V}<-15.53$ have "extra light" compared to the inward extrapolation of a Sersic profile and they attribute this to "wet mergers", consistent with the simulations in [10]. However, when $M_{V}<-21.66$ there is no evidence of recent star formation. Kormendy et al. attributed this to the effect of the hot interstellar medium in providing a working surface for jet outflow. In our view, however, the obstruction of the jet by clouds, is probably a more effective means of confining the jet to the galactic core and inhibiting star formation. The fact that this appears to be more effective in brighter galaxies can possibly be attributed to the greater probability for the occurrence of a radio source in such galaxies. However, this idea needs to be further developed.

\section{References}

[1] D. J. Croton, V. Springel, S. D. M. White, G. De Lucia, C. S. Frenk, L. Gao, A. Jenkins, G. Kauffmann, J. F. Navarro, and N. Yoshida. The many lives of active galactic nuclei: cooling flows, black holes and the luminosities and colours of galaxies. MNRAS, 365:11-28, 2006.

[2] C. M. Booth and J. Schaye. Dark matter haloes determine the masses of supermassive black holes. MNRAS, 405:L1-L5, June 2010.

[3] C. J. Saxton, G. V. Bicknell, R. S. Sutherland, and S. Midgley. Interactions of Jets with Inhomogeneous Cloudy Media. MNRAS, 359:781-800, 2005.

[4] R. S. Sutherland and G. V. Bicknell. Interactions of a Light Hypersonic Jet with a Nonuniform Interstellar Medium. ApJS, 173:37-69, 2007.

[5] A. Y. Wagner and G. V. Bicknell. Relativistic Jet Feedback in Evolving Galaxies. ApJ, 728:29-37, 2011.

[6] G. V. Bicknell, M. A. Dopita, and C. P. O’Dea. Unification of the radio and optical properties of gps and css radio sources. ApJ, 485:112, 1997.

[7] C. Fanti, R. Fanti, D. Dallacasa, R. T. Schilizzi, R. E. Spencer, and C. Stanghellini. Are compact steep spectrum sources young? A\&A, 302:117, 1995.

[8] P. H. N. Nesvadba and M. D. Lehnert. Outflows, Bubbles, and the Role of the Radio Jet: Direct Evidence for AGN Feedback at $z \sim 2$. In C. Charbonnel, F. Combes, \& R. Samadi, editor, SF2A-2008, pages 377, 2008.

[9] J. Kormendy, D. B. Fisher, M. E. Cornell, and R. Bender. Structure and Formation of Elliptical and Spheroidal Galaxies. ApJS, 182:216-309, 2009.

[10] J. C. Mihos and L. Hernquist. Gasdynamics and Starbursts in Major Mergers. ApJ, 464:641, June 1996. 